\title{
The Parameterization Method in Singular Differential-Algebraic Equations ${ }^{\star}$
}

\author{
Vladimir K. Gorbunov and Igor V. Lutoshkin \\ Ulyanovsk State University, L.Tolstoy street 42, \\ 432970 Ulyanovsk, Russia, \\ gvk@vens.ru, LutoshkinIV@ulsu.ru
}

\begin{abstract}
The paper is devoted to the circumstantiation of the parameterization method for classical calculus of variation problems corresponding to the non-linear ODEs. The method is based on a finite parameterization of "control" functions (finitely entering in initial system) and on derivation of the problem functional with respect to control parameters. The first and the second derivatives are calculated with the help of adjoint vector and matrix impulses. The problems of arising degeneration of gradients and optimality conditions of the first order are overcome by using the Newton method. Results of the solution to degenerate DAEs, particularly with non-unique solutions, are presented.
\end{abstract}

\section{Introduction}

In the paper 3] the parameterization method (PM) of optimal control problems was created. This method is based on sequential development of a simple idea to account that as a rule solutions of real optimal control (OC) or calculus of variation $(\mathrm{CV})$ problems have a rather simple structure and control functions can be well approximated in some parametric function class, for example, by splines with moving knots. The PM consists of a finite-dimensional parameterization of control functions. The parameterized variational problem becomes a finitedimensional nonlinear programming (NP) problem whereas its functionals are defined on phase trajectories. Their first and second derivatives on the control parameters may be effectively calculated with the help of variational techniques and adjoint variables. By this the problems of optimization and numerical solution of differential equations are separated. Appropriate parameterization allows one to obtain a NP problem of lower dimension than one obtained under traditional finite-difference approximations.

Another advantage of the PM is a possibility to solve $\mathrm{OC}$ and $\mathrm{CV}$ problems with a continuous admissible control without expansion of the phase space. Continuous control is a natural one in optimization problems of economics dynamic and in variational approach to initial / boundary value problems for differentialalgebraic equations (DAEs), especially in cases of their essential degeneration, when the system has differentiation index [8] more then one or it hasn't a finite index $[12]$.

* Supported by the Russian Foundation for Basic Research, project $N^{o}$ 01-01-00731 
The paper contains reduction schemes for degenerate DAE to the equivalent basic CV problem. The reduced problems as a rule are the degenerate ones with respect to the first order necessary conditions. Also gradients of reduced functionals degenerate near optimal values of parameters. However, effective calculation of degenerate optimization problems can be obtained by means of the second order numerical methods of the Newton type. This general fact was developed for theoretical investigations of degenerate OC problems by R.Gabasov and F.Kirillova (1971) in 2, where the matrix impulse functions, adjoint to the second variations of the optimal trajectories, were introduced. In [9] some degenerate $\mathrm{CV}$ problems were solved thereby using of the second variation of the improved trajectory .

We note here the limited experience of using the second order techniques in variational problems. The PM handles this problem naturally and simply. In difficult unstable problems one can use some regularization of the initial problem [4].

\section{Problem Statement}

Consider the initial / boundary value problem for DAE

$$
\begin{gathered}
\dot{x}=f(x, u(t)), \\
h(x(t), u(t))=0,
\end{gathered}
$$

with conditions

$$
a(x(0), u(0))=0, \quad b(x(T), u(T))=0 .
$$

Here $h(x, u) \in R^{r}, a(x, u) \in R^{m_{1}}, b(x, u) \in R^{m_{2}}, m_{1}+m_{2}=m, n \leq m \leq n+r$.

Difficulties of numerical solution to the problem (1)- (3) appear when the Jacobi matrix of the finite subsystem (2)

$$
\partial h(x(t), u(t)) / \partial u
$$

degenerates on the solution $\{x(t), u(t)\}$. There are some regular numerical methods for the initial value problem for the DAE (11) which have a finite index up to 3 . However, not every system (11), (2) has a finite index and a verification of this property is rather a complex procedure in the common case. The most complex case occurs when the matrix (4) has a variable range on $[0, T][12$.

The problem (11)-(3) is naturally embedded in the class of OC ones with continuous control functions $u(t)$ and can be interpreted as a CV problem. Namely, the problem (1)-(3) is equivalent to the minimization of the functional

$$
J[u]=\int_{0}^{T}\|h(x(t), u(t))\|_{r}^{2} d t+\|b(x(T), u(T))\|_{m_{2}}^{2},
$$

where $\|\cdot\|_{k}$ is the Euclidean norm in $R^{k}$, under conditions

$$
\dot{x}=f(x, u(t)), \quad a(x(0), u(0))=0 .
$$

It can be shown [6], such transform leads to the degenerate CV problem (5), (6). 


\section{Parameterization Method}

Introduce a partition of the interval $[0, T]$

$$
0 \equiv t_{0} \leq t_{1} \leq \ldots \leq t_{N} \equiv T
$$

and define a structure of control functions $u(t)$ on subintervals as

$$
u_{\mu}(t)=u_{\mu}^{k}\left(t ; v_{\mu}^{k}\right), \quad t_{k-1} \leq t<t_{k}, \quad k=1, \ldots, N, \quad \mu=1, \ldots, r .
$$

Here $v_{\mu}^{k} \in R^{d}$, as well as $t_{k}$, are the sought control parameters and $u_{\mu}^{k}\left(t, v_{\mu}^{k}\right)$ are given in analytical form twice-differentiable functions. Further $v^{k}=\left(v_{1}^{k}, \ldots, v_{r}^{k}\right)$.

Conditions (7), (8) present a class of piece-wise continuous control functions with a given number of discontinuities. A class of continuous admissible functions is described by additional conditions $u^{k}\left(t_{k} ; v^{k}\right)=u^{k+1}\left(t_{k} ; v^{k+1}\right)$.

Substituting a control (8) in differential equation (1) we have a solution (its existing is assumed) depended on control parameters

$$
x(t)=z\left(t ; v^{1}, t_{1}, \ldots, v^{k-1}, t_{k-1}, v^{k}\right), \quad t_{k-1} \leq t<t_{k} .
$$

Denote $w^{k}=\left(t_{k}, v^{k}\right) \equiv\left(w_{00}^{k}, w_{11}^{k}, \ldots, w_{r d}^{k}\right)$,

$$
\varphi\left(w^{1}, \ldots, w^{N}\right)=\Phi\left(z\left(T ; w^{1}, \ldots, w^{N-1}, v^{N}\right)\right) .
$$

The problem (5), (6) with the parameterized control (8) is reduced to the finite- dimensional minimization of the function (10) under time-parameter restrictions (7).

Analytical assumptions made above fulfill the conditions of twicedifferentiable continuous dependence of the problem (11), (8) solution on parameters, thus

$$
\frac{\partial \varphi\left(w^{1}, \ldots, w^{N}\right)}{\partial w_{\mu, \alpha}^{k}}=\frac{\partial \Phi(x(T))}{\partial x} \frac{\partial z\left(T ; w^{1}, \ldots, v^{N}\right)}{\partial w_{\mu, \alpha}^{k}}, \quad \mu=0, \ldots, r ; \quad \alpha=0, \ldots, d .
$$

Denote

$$
y^{j \mu \alpha}(t)=\frac{\partial z\left(t ; v^{1}, \ldots, v^{k}\right)}{\partial w_{\mu, \alpha}^{j}}, \quad t_{k-1} \leq t \leq t_{k}, \quad 1 \leq j \leq k \leq N .
$$

These functions are variations of the trajectory (9) on the control parameters. Variations corresponding to parameters $v^{k}$ are solutions of the DE system

$$
\dot{y}^{k \mu \alpha}=\frac{\partial f(x(t), u(t))}{\partial x} y^{k \mu \alpha}+\theta\left(t_{k}-t\right) \frac{\partial f(x(t), u(t))}{\partial u_{\mu}} \frac{\partial u_{\mu}^{k}\left(t ; v^{k}\right)}{\partial v_{\mu, \alpha}^{k}}, t_{k-1} \leq t \leq T
$$

with trivial initial conditions $y^{k \mu \alpha}\left(t_{k-1}\right)=0$. Here the Heaviside function $\theta(t)=$ 0 for $t \leq 0$ and $\theta(t)=1$ for $t>0$. Variations corresponding to the knots $t_{k}$, i.e. functions $y^{k 00}(t)$, are defined on $\left[t_{k}, T\right]$ by the similar homogeneous DE system 
with initial condition $y^{k 00}\left(t_{k}\right)=f\left(x\left(t_{k}\right), u^{k}\left(t_{k} ; v^{k}\right)\right)-f\left(x\left(t_{k}\right), u^{k+1}\left(t_{k} ; v^{k+1}\right)\right)$. If admissible controls are continuous, then the right hand of the condition equals to zero, correspondingly, $y^{k 00}(t) \equiv 0$.

So, one can calculate the derivatives (11) and apply the gradient method for the finite-dimensional minimization problem. However the such scheme is rather complicated because the number of variations equals the number of control parameters. It is essentially simplified via using adjoint functions.

Introduce the Hamilton-Pontrjagin function $H(p, x, u)=\sum_{i=1}^{n} p_{i} f_{i}(x, u)$, the vector function $p(t)$ on $[0, T]$ (adjoint one to the first variations (12))

$$
\dot{p}=-\frac{\partial H(p, x(t), u(t))}{\partial x}, \quad p(T)=\frac{\partial \Phi(x(T))}{\partial x},
$$

and the function $M(t)=H(p(t), x(t), u(t))$.

The first derivatives (11) can be calculated [3] by formulas:

$$
\begin{aligned}
& \frac{\partial \varphi\left(w^{1}, \ldots, w^{N}\right)}{\partial t_{k}}=M\left(t_{k}-0\right)-M\left(t_{k}+0\right), \quad 1 \leq k \leq N-1 ; \\
& \frac{\partial \varphi}{\partial v_{\mu \alpha}^{k}}=\int_{t_{k-1}}^{t_{k}} \frac{\partial H\left(p(t), x(t), u^{k}\left(t ; v^{k}\right)\right)}{\partial u_{\mu}} \frac{\partial u_{\mu}^{k}\left(t ; v^{k}\right)}{\partial v_{\mu \alpha}^{k}} d t, 1 \leq k \leq N .
\end{aligned}
$$

So, in order to calculate the derivatives (11) only one additional Cauchy problem, defined adjoint function $p(t)$, should be solved.

In the considering case control functions $u(t)$ are continuous hence derivatives $\partial \varphi / \partial t_{k}$, as well as variations $y^{k 00}(t)$, are equal to zero.

The second derivation of (11) yields $(0 \leq \mu, \nu \leq r ; 0 \leq \alpha, \beta \leq d ; 1 \leq$ $j, k \leq N)$

$$
\frac{\partial^{2} \varphi\left(w^{1}, \ldots, w^{N}\right)}{\partial w_{\nu, \beta}^{k} \partial w_{\mu, \alpha}^{j}}=y^{j \mu \alpha}(T) \frac{\partial^{2} \Phi(x(T))}{\partial x^{2}} y^{k \nu \beta}(T)+\frac{\partial \Phi(x(T))}{\partial x} \frac{\partial^{2} z\left(T ; w^{1}, \ldots, v^{N}\right)}{\partial w_{\nu, \beta}^{k} \partial w_{\mu, \alpha}^{j}} .
$$

The second variations $\partial^{2} z / \partial w_{\nu, \beta}^{k} \partial w_{\mu, \alpha}^{j}$ as well as the first (12) are defined by some Cauchy problems 311]. It resolves, in principle, the problem of the second derivatives (13) calculation and using the Newton method. But the calculations also essentially simplified via using appropriate adjoint functions. These functions are the matrix impulses $\Psi(t)$ defined in [2] by the problem

$$
\begin{gathered}
\dot{\Psi}=-\left[\frac{\partial f(x(t), u(t))}{\partial x}+\left(\frac{\partial f(x(t), u(t))}{\partial x}\right)^{T}\right] \Psi-\frac{\partial^{2} H(p(t), x(t), u(t))}{\partial x^{2}}, \\
\Psi(T)=\frac{\partial^{2} \Phi(x(T))}{\partial x^{2}} .
\end{gathered}
$$

The second derivatives of an admissible trajectory of the system (1) on control parameters in the case of continuous control can be calculated by the next expressions: 


$$
\begin{gathered}
\frac{\partial^{2} \varphi\left(w^{1}, \ldots, w^{N}\right)}{\partial v_{\mu, \alpha}^{j} \partial v_{\nu, \beta}^{k}}=\int_{t_{k-1}}^{t_{k}}\left\{\frac { \partial u _ { \nu } ^ { k } ( t ; v ^ { k } ) } { \partial v _ { \nu , \beta } ^ { k } } \left[\frac{\partial^{2} H(p(t), x(t), u(t))}{\partial u_{\nu} \partial x}+\right.\right. \\
\left.\left(\frac{\partial f(x(t), u(t))}{\partial u_{\nu}}\right)^{T} \Psi(t)\right] y^{j \mu \alpha}(t)+\delta_{j k}\left[\frac{\partial u_{\nu}^{k}\left(t ; v^{k}\right)}{\partial v_{\nu, \beta}^{k}} \frac{\partial u_{\mu}^{k}\left(t ; v^{k}\right)}{\partial v_{\mu, \alpha}^{k}} \frac{\partial^{2} H(p(t), x(t), u(t))}{\partial u_{\nu} \partial u_{\mu}}+\right. \\
\frac{\partial u_{\mu}^{k}\left(t ; v^{k}\right)}{\partial v_{\mu, \alpha}^{k}}\left[\frac{\partial^{2} H(p(t), x(t), u(t))}{\partial u_{\mu} \partial x}+\left(\frac{\partial f(x(t), u(t))}{\partial u_{\mu}}\right)^{T} \Psi(t)\right] y^{k \nu \beta}(t)+ \\
\left.\left.\delta_{\mu \nu} \frac{\partial^{2} u_{\nu}^{k}\left(t ; v^{k}\right)}{\partial v_{\mu, \alpha}^{k} \partial v_{\nu, \beta}^{k}} \frac{\partial H(p(t), x(t), u(t))}{\partial u_{\nu}}\right]\right\} t, \quad j \leq k ; \\
\frac{\partial^{2} \varphi\left(w^{1}, \ldots, w^{N}\right)}{\partial v_{\mu, \alpha}^{j} \partial t_{k}}=\delta_{j k} \frac{\partial u_{\mu}^{k}\left(t_{k}\right)}{\partial v_{\mu, \alpha}^{k}} \frac{\partial H\left(p\left(t_{k}\right), x\left(t_{k}\right), u^{k}\left(t_{k} ; v^{k}\right)\right)}{\partial u_{\mu}}, j \leq k \\
\frac{\partial^{2} \varphi\left(w^{1}, \ldots, w^{N}\right)}{\partial t_{j} \partial v_{\nu, \beta}^{k}}=-\delta_{j(k-1)} \frac{\partial H\left(p\left(t_{k-1}\right), x\left(t_{k-1}\right), u^{k}\left(t_{k-1} ; v^{k}\right)\right)}{\partial u_{\nu}}, \quad j<k \\
\frac{\partial^{2} \varphi\left(w^{1}, \ldots, w^{N}\right)}{\partial t_{j} \partial t_{k}}=\delta_{j k}\left(\frac{\partial H\left(p\left(t_{k}\right), x\left(t_{k}\right), u^{k}\left(t_{k} ; v^{k}\right)\right)}{\partial u} \frac{\partial u^{k}\left(t_{k} ; v^{k}\right)}{\partial t}-\right. \\
\partial t
\end{gathered}
$$

These formulas are a particular case of more complicate ones for the class of piece-wise continuous admissible control [5]11].

So the calculations of the second derivatives (13) require solution of a few Cauchy problems for the first variations $y(t)$ and the adjoint variables $p(t), \Psi(t)$. It allows ones to apply the Newton method on the base of the penalty function one for the parameterized functional (10) minimization under conditions (7). Note, the variational problem (5), (6), corresponding to the initial problem for DAE (11), can be solved sequentially on sufficiently small intervals $\left[t_{k-1}, t_{k}\right]$. The control function components $u_{i}(t)$ can be assigned as square or cubic parabolas. The obtained control will be a polynomial spline.

\section{Numerical Example}

Example 1. Let's consider on the interval $[0 ; T]$ a DAE ([10 1])

$$
\begin{gathered}
\dot{x}_{1}=10 t \exp \left(5 u_{2}(t)-1\right) x_{2}(t), \quad \dot{x}_{2}=-2 t \ln \left(u_{1}(t)\right), \\
u_{1}(t)=x_{1}(t)^{1 / 5}, \quad u_{2}(t)=\left(x_{2}^{2}(t)+u_{2}^{2}(t)\right) / 2 .
\end{gathered}
$$

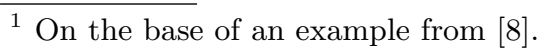


One of this nonlinear system's solutions is

$$
\begin{gathered}
\bar{x}_{1}(t)=\exp \left(5 \sin \left(t^{2}\right)\right), \quad \bar{x}_{2}(t)=\cos \left(t^{2}\right), \\
\bar{u}_{1}(t)=\exp \left(\sin \left(t^{2}\right)\right), \quad \overline{u_{2}}(t)=\sin \left(t^{2}\right)+1 .
\end{gathered}
$$

We state here the initial problem corresponding to the solution. Moreover, it is desire to construct numerically all solutions on the interval under given $T$.

This problem is a base test in some articles of the author [10], but on the interval $[1.0708712 ; 1.423836]$. The cause is in degeneration of the Jacobi matrix of the finite subsystem

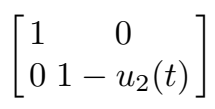

in points $t_{k}$ where $u_{2}(t)=1$. Such points, corresponding to the solution (E2), are $t_{k}=\sqrt{k \pi}(k=0,1, \ldots)$.

Solutions of (E1) bifurcate at the points and the system isn't reducible to normal Cauchy form on corresponding intervals, i.e. it isn't a finite index system and it can't be solved by known methods developed for DAE of finite index, particularly, by the methods of [10], assigned to DAE of index one.

The character of degenerating the second finite restriction points at existing two implicit functions $u_{2}^{ \pm}\left(x_{2}\right)=1 \pm \sqrt{1-x_{2}^{2}}$ at a vicinity of initial conditions $x_{2}(0)=1, u_{2}(0)=1$. So, at the initial point two solution are generated.

To construct numerically two solutions, introduce the functional

$$
\begin{aligned}
& J_{\alpha}[u]=\int_{0}^{T}\left[\left(u_{2}(t)-\left(x_{2}^{2}(t)+u_{2}^{2}(t)\right) / 2\right)^{2}+\right. \\
& \left.\left(x_{1}(t)^{1 / 5}-u_{1}(t)\right)^{2}+\alpha\left(u_{2}(t)-z(t)\right)^{2}\right] d t .
\end{aligned}
$$

Here $\alpha \geq 0, z(t)$ is a trial function presented a priori properties of the solution component $u_{2}(t)$. Consider the problem to minimize $(E 3)$ under restrictions

$$
\begin{aligned}
& \dot{x}_{1}=10 t \exp \left(5 u_{2}(t)-1\right) x_{2}, \quad \dot{x}_{2}=-2 t \ln \left(u_{1}(t)\right) \\
& x_{1}(0)=x_{2}(0)=1
\end{aligned}
$$

This problem is equivalent to the initial one under $\alpha=0$ and if $\alpha>0$ it approximates a solution of (E1) which has the component $u_{2}(t)$ similar to $z(t)$. Having such solution depending on $\alpha$ we use it as initial approximation for a repeating minimization (E3) under $\alpha=0$.

The problem (E3), (E4) was solved such a way with $T=0.5, z(t)=1+\sin (t)$ and $z(t)=1-\sin (t)$ consequently on 5 equal intervals $[0 ; 0.1], \ldots,[0.4 ; 0.5]$. Every problem was solved in the class of square parabolic "controls" $u_{i}(t)$. The corresponding Cauchy problems were integrated by the 4-th order Runge-Kutta method with step 0.001 .

At the case $z(t)=1+\sin (t)$ the obtained approximation $\left(x^{+}, u^{+}\right)$of solution (E2) provides $J_{0}\left[u^{+}\right]=4.7 \times 10^{-11}$ and maximal coordinate deviations are correspondingly $\left(4.7 \times 10^{-5} ; 8.4 \times 10^{-7} ; 3.9 \times 10^{-5} ; 2.1 \times 10^{-4}\right)$. 
At the case $z(t)=1-\sin (t)$ there was obtained another approximation $\left(x^{-}, u^{-}\right)$with unknown analytical expression. Here $J_{0}\left[u^{-}\right]=1.6 \times 10^{-10}$. The $x_{1}^{-}(t)$ is increasing to the value $x_{1}^{-}(0.5)=1.77$ whereas $x_{1}^{+}(0.5)=3.45$. The $u_{2}^{-}(t)$ is diminishing to the value $u_{2}^{-}(0.5)=0.81$ whereas $u_{2}^{+}(t)$ is increasing to $u_{2}^{+}(0.5)=1.25$.

Example 2. Let's consider singular linear DE system ([1]).

$$
A(t) \dot{x}-B(t) x(t)=f(t),
$$

where

$$
\begin{gathered}
A(t)=\left[\begin{array}{cccc}
t^{2} & 2 t^{2} & t^{2} & t \\
t^{2} & t^{2} & 2 t^{2} & t \\
t^{2} & t^{2} & t^{2} & t \\
0 & 0 & 0 & 0
\end{array}\right], \quad B(t)=\left[\begin{array}{cccc}
3 & 3 t & t & 0 \\
t & 3 & 3 t & 0 \\
t & t & 3 & 0 \\
t & t & t & 1
\end{array}\right], \\
f(t)=\left[\begin{array}{c}
4 t^{2}-3 t-3 \\
4 t^{2}-3 t-3 \\
3 t^{2}-t-3 \\
-3 t-1
\end{array}\right] \exp (t),
\end{gathered}
$$

on the interval $[0 ; 1]$, with the initial condition $x(0)=(1,1,1,1)^{T}$, corresponding to the solution

$$
x(t)=(1,1,1,1)^{T} \exp (t) .
$$

On the $[0 ; 1]$ the matrix $A(t)$ has a variable range, which equals zero $(t=0)$ or three $(t>0)$. Well-known methods are inapplicable for this problem. In the paper 12] the problem of solution existence for such kind degenerate (singular) equations was considered. In the [1] an analogous problem was numerically solved by the special collocation-difference method on the interval $[1 ; 2]$.

The considered DE problem is linear and the initial problem was solved by the normal spline collocation method in the 7] on the [0;1]. To solve this problem on the $[0 ; 1]$ by the parameterization method it was reduced to $\mathrm{VC}$ problem:

$$
\dot{x}=u(t)
$$

with initial condition $x(0)=(1,1,1,1)^{T}$ and minimizing the functional

$$
J\left(u ; t_{0}, T\right)=\int_{t_{0}}^{T}\|A(t) u(t)-B(t) x(t)-f(t)\|^{2} d t,
$$

where $t_{0}=0, T=1$.

Clearly, the functional $J\left(u ; t_{0}, T\right)$ is additive, so it can be solved consequently on the partial intervals $\left[t_{k}, t_{k+1}\right]$, which are a partition of the interval $\left[t_{0}, T\right]$. Here the functionals $J\left(u ; t_{k}, t_{k+1}\right)$ are minimized consequently.

The initial problem was solved consequently on the five equal intervals $[0 ; 0,2],[0,2 ; 0,4], \ldots,[0,8 ; 1,0]$ by two ways. In the first way it was used square parabolas for the approximation of the control $u(t)$ :

$$
u_{i}(t)=a_{i} * t^{2}+b_{i} * t+c_{i}, \quad i=1,2,3,4 .
$$


In the second way exponential functions

$$
u_{i}(t)=a_{i} * \exp \left(b_{i} * t\right), \quad i=1,2,3,4,
$$

were used.

The Cauchy problems were integrated by the second order Runge-Kutta method. The corresponding NP problems were solved at first by the gradient method, then by the Newton one. The obtained errors (the maximum deviation of components on the integration net) for different steps of integration $h$ are presented in the table 1.

\section{Table 1.}

\begin{tabular}{|c|c|c|c|}
\hline$h$ & 0,01 & 0,005 & 0,001 \\
\hline way 1 & 0,0056 & 0,017 & 0,018 \\
\hline way 2 & $4.0 \times 10^{-4}$ & $2.6 \times 10^{-5}$ & $2,4 \times 10^{-8}$ \\
\hline
\end{tabular}

These results show the importance of using of adequate a priori representation of seeking solution. Besides we should mark paradoxical increase of an error with a diminution of an integration step in the way 1 . It can be explained by accumulation of round-off errors, as the usual arithmetic of double precision was used.

\section{Conclusion}

So, these examples demonstrate a capacity for applying the parameterization method of the second order in the standard form for solving singular DE problems. Success of the PM application to degenerate DA systems can be explained by picking out the regular differential subsystem and fixing the structure of the control solution components on the integration intervals. Moreover, these intervals are more much then a step of a difference scheme that was applied to the regular Cauchy problems.

\section{References}

1. Bojarincev, Ju.E., Danilov, V.A., Loginov, A.A., Chistyakov, V.F.: Numeric Methods of Solving Singular Systems. Nauka, Novosibirsk (1989)

2. Gabasov, R., Kirillova, F.: Qualitative theory of optimal processes. Nauka, Moscow (1971) (Russian)

3. Gorbunov, V.K.: The parameterization method for optimal control problems. Computational Mathematics and Mathematical Physics. Vol. 19. 2 (1979)

4. Gorbunov, V.K.: Regularization of degenerate equations and inequalities under explicit data parameterization. J. Inv. Ill-Posed Problems. Vol. 9. 6 (2001) 575594 
5. Gorbunov, V.K., Lutoshkin, I.V.: Second derivatives of parameterized optimal control problems. Proceedings of 11-th Baikal International School-Seminar. Irkutsk 4 (1998) 90-93

6. Gorbunov, V.K., Lutoshkin, I.V.: Development and Experience of Applying the Parameterization Method in Degenerate Problems of Dynamical Optimization. J. of Computer and Systems Sciences International. (2003) (to appear)

7. Gorbunov, V.K., Petrischev, V.V.: Development of the normal collocation spline method for linear differential equations. Comput. Math. and Math. Phys. Vol. 43. (2003) (to appear)

8. Hairer, E., Wanner, G.: Solving Ordinary Differential equations II: Stiff and Differential-algebraic Problems. Springer-Verlag, Berlin (1996)

9. Henrion, R.: La théorie de la variation seconde et ses applications en commande optimale. Bruxelles-Palais des Academies, Bruxelles (1974)

10. Kulikov, G.Yu.: Numerical solution of the Cauchy problem for a system of differential-algebraic equations with the use of implicit Runge-Kutta methods with a untrivial predictor. Comput. Math. and Math. Phys. Vol. 38. 1 (1998)

11. Lutoshkin, I.V.: Using the parameterization method in degenerate problems. Thesis of Ph.D., Phys.-Math. Ulyanovsk (2000) (Russian)

12. März, R., Weinmüller: Solvability of Boundary value problems for systems of singular differential-algebraic equations. SIAM J. Math. Anal. Vol. 24.1 (1993) 200-215 